\title{
On communication over an entanglement-assisted quantum channel
}

\author{
Ashwin Nayak \\ Computer Science Department, and \\ Institute for Quantum Information \\ California Institute of Technology \\ Pasadena, CA 91125-8000 \\ nayak@cs.caltech.edu
}

\author{
Julia Salzman ${ }^{\dagger}$ \\ Mathematics Department \\ Princeton University \\ Fine Hall, Washington Road \\ Princeton, NJ 08544-1000 \\ jsalzman@princeton.edu
}

\begin{abstract}
Shared entanglement is a resource available to parties communicating over a quantum channel, much akin to public coins in classical communication protocols. Whereas shared randomness does not help in the transmission of information, or significantly reduce the classical complexity of computing functions (as compared to private-coin protocols), shared entanglement leads to startling phenomena such as "quantum teleportation" and "superdense coding."

The problem of characterising the power of prior entanglement has puzzled many researchers. In this paper, we revisit the problem of transmitting classical bits over an entanglement-assisted quantum channel. We derive a new, optimal bound on the number of quantum bits required for this task, for any given probability of error. All known lower bounds in the setting of bounded error entanglementassisted communication are based on sophisticated information theoretic arguments. In contrast, our result is derived from first principles, using a simple linear algebraic technique.
\end{abstract}

\section{Categories and Subject Descriptors}

F.2 [Theory of Computation]: Analysis of Algorithms and Problem Complexity

\section{General Terms}

Theory

\footnotetext{
${ }^{*}$ Supported by Charles Lee Powell Foundation, and NSF grants CCR 0049092 and EIA 0086038.

${ }^{\dagger}$ A part of this work was done while this author was visiting Caltech on a Summer Undergraduate Research Fellowship.
}

Permission to make digital or hard copies of all or part of this work for personal or classroom use is granted without fee provided that copies are not made or distributed for profit or commercial advantage and that copies bear this notice and the full citation on the first page. To copy otherwise, to republish, to post on servers or to redistribute to lists, requires prior specific permission and/or a fee.

STOC'02, May 19-21, 2002, Montreal, Quebec, Canada.

Copyright 2002 ACM 1-58113-495-9/02/0005 ...\$5.00.

\section{Keywords}

Quantum communication, entanglement-assisted quantum channel, communication complexity

\section{INTRODUCTION}

Consider two parties solving a distributed task by communicating with each other. Remarkably, it has been shown that if the two parties are equipped with a quantum computer and can communicate by exchanging quantum states, they can solve certain tasks at a significantly smaller communication cost, when compared to classical protocols [6, 17, 18. This is especially surprising since an early result due to Holevo 10 (later explained in simpler terms by Nayak 16]) rules out obvious methods of compressing classical information into succinct quantum messages - Holevo's theorem implies that $n$ quantum bits of communication are necessary to transmit $n$ classical bits of information.

An additional resource that is available to parties communicating over a quantum channel is "shared entanglement": the two parties may be given some number of quantum bits jointly prepared in a fixed superposition, prior to communicating with each other. For example, they may jointly hold some number of EPR pairs. If The quantum channel is then said to be "entanglement-assisted."

Shared randomness does not help in the transmission of information from one party to another, or significantly reduce the classical complexity of computing functions visa-vis private-coin protocols [14, Section 3.3]. On the other hand, prior entanglement leads to startling phenomena such as "quantum teleportation" 22 and "superdense coding" [4. In particular, superdense coding allows us to transmit $n$ classical bits with perfect fidelity by sending only $n / 2$ quantum bits. The problem of characterising the power of prior entanglement has baffled many researchers 17,12 , especially in the setting of bounded-error protocols. It is open whether it leads to more than a factor of two savings (using superdense coding) or more than an additive $O(\log n)$ savings (when used to create shared randomness). Few lower bounds are known for communication problems in this setting [8, 15, 11, 13, and are all derived using sophisticated informationtheoretic techniques.

In this paper, we focus on the most basic problem in

\footnotetext{
${ }^{1}$ An EPR pair consists of two qubits prepared in the maximally entangled state $\frac{1}{\sqrt{2}}(|00\rangle+|11\rangle)$.
} 
the setting of communication over an entanglement-assisted quantum channel, that of transmitting classical bits from one party to another. We derive optimal bounds on the number of quantum bits required for this task, for any given probability of error.

Theorem 1.1. Suppose one party, Alice, wishes to communicate $n$ bits to the other, Bob, over an entanglementassisted quantum channel. For any choice of the entangled state, and any protocol such that the total number of qubits sent by Alice to Bob (over all the rounds of communication) is $m_{A}$, let $Y$ be the random variable denoting Bob's output, when Alice wishes to convey $X$. If $X$ is distributed uniformly over $\{0,1\}^{n}$, the probability that Bob correctly recovers $X$ is is bounded as

$$
\operatorname{Pr}[Y=X] \leq \frac{2^{2 m_{A}}}{2^{n}}
$$

irrespective of the number of qubits sent by Bob to Alice, or the number of rounds of communication.

Thus, for protocols with probability of success $\delta>0$, we get the optimal lower bound of $m_{A} \geq \frac{1}{2}\left(n-\log \frac{1}{\delta}\right)$. This improves over the lower bound of $m_{A} \geq \frac{1}{2}(\delta n-H(\delta))$ implied by a result of Cleve et al. [8, Theorem 2], when combined with Fano's inequality [9, Section 2.11].

REMARK: A bound for non-uniform distributions over the inputs also follows from the proof of Theorem 1.1, as in [16]. Note also that an optimal bound of $n-\log \frac{1}{\delta}$ for the total number of quantum bits exchanged, including the communication required to create the prior entanglement, is implied by [16, Theorem 2.4].

All known lower bounds for bounded-error communication using prior entanglement are based on complex informationtheoretic arguments. In fact, one might be lead to believe that such techniques are inevitable - any lower bound proof necessarily depends on the property that the prior shared state contains no information about the inputs. Contrary to this, our results are derived from first principles, using a linear algebraic technique that has its roots in the work of Nayak 16]. In order to prove Theorem 1.1, we give a new characterisation of the joint state at the end of a quantum protocol that complements the characterisation due to Yao [20]. It greatly clarifies the role of shared entanglement in communication, and we expect that it will further enhance our conceptual understanding of quantum communication.

Putting Theorem 1.1 together with a reduction due to Cleve et al. [8], we get a new lower bound of $\frac{1}{2}\left(n-2 \log \frac{1}{1-2 \epsilon}\right)$ for the $\epsilon$-error entanglement-assisted communication complexity of the inner product function. The previous best lower bound was $\frac{1}{2}\left((1-2 \epsilon)^{2} n-1\right)$ due to [8]. Since there is a classical $n-\log \frac{1}{1-2 \epsilon}+1$ bit public-coin protocol for Inner Product, and hence a $\frac{1}{2}\left(n-\log \frac{1}{1-2 \epsilon}+1\right)$ qubit quantum protocol with shared EPR pairs, our lower bound is nearoptimal. Our results thus provide more examples where shared entanglement leads to at most a factor of two savings in communication.

The lower bound of $\frac{1}{2}\left(n-2 \log \frac{1}{1-2 \epsilon}\right)$ for Inner Product stated above was independently discovered by van Dam and Hayden [19] in the case of communication with shared EPR pairs. However, they follow an information-theoretic approach that provably breaks down in the presence of arbitrary prior entanglement.

\subsection{Organisation of the paper}

The quantum communication model, and the associated terminology and notation are described in Section 2. We begin by analysing quantum encoding of classical bits in the presence of entanglement in Section 3. In fact, we first consider a very restricted kind of encoding, where the shared state consists of EPR pairs, and no ancillary qubits are used in the encoding (Section 3.1). This contains the basic elements of the proof for general encoding as well, which is the subject of Section 3.2. Building on the insight gained from the study of quantum encoding, we extend our results to the case of interactive communication in Section 4 .

\section{PRELIMINARIES}

\subsection{The communication model}

In the quantum communication model of Yao [20], two parties Alice and Bob hold qubits. When the game starts Alice holds a superposition $|x\rangle$ and Bob holds $|y\rangle$, representing the input to the two players. The initial joint state is thus $|x\rangle_{A} \otimes|y\rangle_{B}$, where a subscript indicates the player holding that set of qubits. Furthermore each player has an arbitrarily large supply of private qubits in some fixed basis state, say $|\overline{0}\rangle$. The two parties then play in turns. Suppose it is Alice's turn to play. Alice can do an arbitrary unitary transformation on her qubits and then send one or more qubits to Bob. Sending qubits does not change the overall superposition, but rather changes the ownership of the qubits, allowing Bob to apply his next unitary transformation on the newly received qubits. At the end of the protocol, one player measures one or more qubits in some basis, and declares those as the result of the protocol. (In cases where a specific player is required to know the answer, that player makes the measurement.) In a classical probabilistic protocol the players may only exchange messages composed of classical bits.

Note that there is no loss of generality in not allowing the players to measure a subset of their quantum bits in the intermediate steps of a protocol. This is because all measurements may be postponed to the end by the principle of safe storage [5. We also assume, w.l.o.g., that the players do not modify the state of the qubits containing their inputs.

In the classical model we can also define a public-coin version, in which the players are also allowed to access a shared source of random bits without any communication cost. The classical public and private-coin models are strongly related (see 14. Section 3.3]). In the quantum analogue of the public-coin model, Alice and Bob may initially share an arbitrary number of quantum bits which are in some pure state that is independent of the inputs. This is known as communication with prior entanglement [8, 7, or in informationtheoretic terms, as communication over an entanglementassisted quantum channel 3].

The complexity of a quantum (or classical) protocol is the number of qubits (respectively, bits) exchanged between the two players. We say a protocol computes a function $f: X \times$ $Y \mapsto\{0,1\}$ with $\epsilon \geq 0$ error if, for any input $x \in X, y \in Y$, the probability that the two players compute $f(x, y)$ is at least $1-\epsilon . Q_{\epsilon}(f)$ (resp. $R_{\epsilon}(f)$ ) denotes the complexity of the best quantum (resp. probabilistic) protocol that computes $f$ with at most $\epsilon$ error. We will use the notation $Q_{\epsilon}^{*}(f)$ for entanglement-assisted quantum communication of the function $f$. 
On occasion, we will concentrate on communication in one round, since this often sheds light on fundamental properties of protocols for certain problems. The message in a oneround protocol in which only one player gets an input is called an encoding of the input. The operations done by the other player, and her measurement are together referred to as decoding.

\subsection{Miscellanea}

A mixed state over a set of qubits is a probability distribution $\left\{p_{i},\left|\phi_{i}\right\rangle\right\}$ over superpositions (or pure states), where the state $\left|\phi_{i}\right\rangle$ occurs with probability $p_{i}$. We will sometimes use the notation $\left\{\left|\phi_{i}\right\rangle\right\}$ for a mixed state, where the states $\left|\phi_{i}\right\rangle$ are in general unnormalised, and are such that $\sum_{i}\left\|\phi_{i}\right\|^{2}=$ 1.

The following theorem gives a useful characterisation of bi-partite quantum states (see [17, Section 2.5]).

TheOREM 2.1 (SCHMIDT DECOMPOSITION THEOREM). Any unit vector $|\phi\rangle$ in a bi-partite Hilbert space $\mathcal{H} \otimes \mathcal{K}$ may be represented as

$$
|\phi\rangle=\sum_{i} \sqrt{\lambda_{i}}\left|e_{i}\right\rangle\left|f_{i}\right\rangle
$$

where $\left\{\left|e_{i}\right\rangle\right\}$ and $\left\{\left|f_{i}\right\rangle\right\}$ are orthonormal sets of states in $\mathcal{H}$ and $\mathcal{K}$ respectively, and the $\lambda_{i} \geq 0$ are such that $\sum_{i} \lambda_{i}=1$.

We denote the identity operator on states over $k$ qubits by $I_{k}$.

\section{BOUNDS FOR ENCODING}

In this section we concentrate on one-way protocols, or encoding, by which one party, Alice, wishes to send some number of classical bits to Bob.

\subsection{Encoding over EPR pairs, without ancilla}

We first prove our results in the case where Alice does not use any ancillary qubits in the encoding process, and Alice and Bob share some number of EPR pairs. This motivates the proof in the more general case, and illustrates its essential elements.

We start with a simple property of maximally entangled states, such as EPR pairs. This allows us to analyse the encoding process easily.

LEMMA 3.1. For any unitary transformation $U$ on $E q u$ bits, and any orthonormal set $\left\{\left|\phi_{a}\right\rangle: a \in\{0,1\}^{E}\right\}$ over $E^{\prime} \geq$ E qubits,

$$
\sum_{a \in\{0,1\}^{E}} U|a\rangle\left|\phi_{a}\right\rangle=\sum_{a \in\{0,1\}^{E}}|a\rangle \tilde{U}\left|\phi_{a}\right\rangle,
$$

where $\tilde{U}$ is any transformation on $E^{\prime}$ qubits such that for all $a, a^{\prime} \in\{0,1\}^{E},\left\langle\phi_{a}|\tilde{U}| \phi_{a^{\prime}}\right\rangle=\left\langle a^{\prime}|U| a\right\rangle$.

Proof. Observe that for $b, c \in\{0,1\}^{E}$,

$$
\begin{aligned}
\left\langle b\left|\left\langle\phi_{c}\left|\sum_{a \in\{0,1\}^{E}} U\right| a\right\rangle\right| \phi_{a}\right\rangle & =\langle b|U| c\rangle \\
& =\left\langle\phi_{c}|\tilde{U}| \phi_{b}\right\rangle \\
& =\left\langle b\left|\left\langle\phi_{c}\left|\sum_{a \in\{0,1\}^{E}}\right| a\right\rangle \tilde{U}\right| \phi_{a}\right\rangle .
\end{aligned}
$$

The lemma follows.
We can now characterise the encoding process (without ancilla) as follows.

LEMMA 3.2. Suppose that Alice performs a unitary transformation on her share of E EPR pairs, and then sends $m$ of the $E$ qubits to Bob. Then, Bob has $E+m$ qubits in a mixed state that can be represented as $\left\{p_{l},\left|\phi_{l}\right\rangle\right\} \quad\left(l \in\{0,1\}^{E-m}\right)$ with $\left\{\left|\phi_{l}\right\rangle\right\}_{l}$ orthonormal, and $p_{l}=\frac{1}{2^{E-m}}$.

Proof. Suppose that Alice applies a transformation $V$ to her part of the state. By Lemma 3.1, the resulting state is

$$
\frac{1}{2^{E / 2}} \sum_{a \in\{0,1\}^{E}} V|a\rangle_{A}|a\rangle_{B}=\frac{1}{2^{E / 2}} \sum_{a \in\{0,1\}^{E}}|a\rangle_{A} V^{\top}|a\rangle_{B} .
$$

After the communication, Alice and Bob's joint state may be written as (w.l.o.g., Alice sends the rightmost $m$ qubits to Bob):

$$
\frac{1}{2^{(E-m) / 2}} \sum_{l \in\{0,1\}^{E-m}}|l\rangle_{A} \frac{1}{2^{m / 2}} \sum_{r \in\{0,1\}^{m}}|r\rangle_{B} V^{\top}|l r\rangle_{B} .
$$

Consider the mixed state on Bob's side obtained when Alice measures her qubits in the standard basis. The probability $p_{l}$ of Alice observing any given $l$ is $\frac{1}{2^{E-m}}$. The state of Bob's $E+m$ qubits when Alice gets outcome $l$ is

$$
\left|\phi_{l}\right\rangle=\frac{1}{2^{m / 2}} \sum_{r \in\{0,1\}^{m}}|r\rangle V^{\top}|l r\rangle .
$$

We may easily verify that these are orthonormal for different $l$ :

$$
\begin{aligned}
\left\langle\phi_{l} \mid \phi_{l^{\prime}}\right\rangle & =\frac{1}{2^{m}} \sum_{r}\left\langle l r\left|V^{*} V^{\top}\right| l^{\prime} r\right\rangle \\
& =\frac{1}{2^{m}} \sum_{r}\left\langle l r \mid l^{\prime} r\right\rangle \\
& =\delta_{l, l^{\prime}} .
\end{aligned}
$$

Note that the above measurement by Alice does not affect the decoding process; Bob's density matrix remains unchanged by it (see [17, Section 2.4], especially Section 2.4.3). Nonetheless, it allows us to express Bob's mixed state in a convenient form.

This proves the lemma.

By a simple dimensional argument, can now get an alternative proof of the fact that the superdense coding scheme of [- 1 is optimal (in the case of encoding without ancilla). We omit the proof.

In general, we can tolerate a little error in the decoding process. This opens up the possibility of Alice being able to reduce the communication significantly. The following theorem places limits on the savings achieved.

TheOREM 3.3. If Alice encodes messages $x \in\{0,1\}^{n}$ over EPR pairs without ancilla, and sends $m$ qubits to Bob, the probability of correct decoding of a message chosen uniformly at random is bounded as $\operatorname{Pr}[$ correct decoding $] \leq \frac{2^{2 m}}{2^{n}}$.

Proof. Suppose that the number of EPR pairs Alice and Bob share initially is $E$. Let $\left\{p_{x, l},\left|\phi_{x, l}\right\rangle\right\}_{l}$ be Bob's 
mixed state when Alice has input $x \in\{0,1\}^{n}$, as given by Lemma 3.2.

We may view the entire decoding procedure used by Bob as measuring the encoded state with some ancillary qubits (w.l.o.g., assumed to be initialised to state $|\overline{0}\rangle$ ) with the projection operators $\left\{P_{y}\right\}$. Here, the outcome $y \in\{0,1\}^{n}$ corresponds to Bob's guess for the encoded message. We will omit the ancilla from the expressions below, for clarity of exposition.

Let $C$ be the event that Bob decodes a message correctly, $C_{x}\left(C_{x, l}\right)$ that he does so on receiving the encoding of $x$ $\left(\left|\phi_{x, l}\right\rangle\right.$, respectively). Let $x$ be the event that Alice encodes message $x$, and $x_{l}$ that $\left|\phi_{x, l}\right\rangle$ is prepared given that $x$ is encoded. Then

$$
\begin{aligned}
\operatorname{Pr}[C] & =\sum_{x} \operatorname{Pr}\left[C_{x}\right] \cdot \operatorname{Pr}[x] \\
& =\sum_{x} \frac{\operatorname{Pr}\left[C_{x}\right]}{2^{n}} \\
& =\sum_{x, l} \frac{\operatorname{Pr}\left[C_{x, l}\right] \cdot \operatorname{Pr}\left[x_{l}\right]}{2^{n}} \\
& =\sum_{x, l} \frac{\operatorname{Pr}\left[C_{x, l}\right]}{2^{E-m} 2^{n}} .
\end{aligned}
$$

It thus suffices to bound $\sum_{x, l} \operatorname{Pr}\left[C_{x, l}\right]$. Observe that

$$
\operatorname{Pr}\left[C_{x, l}\right]=\| P_{x}\left|\phi_{x, l}\right\rangle \|^{2} .
$$

We introduce some notation. For each $x$, let $H_{x}$ be the space spanned by $\left\{\left|\phi_{x, l}\right\rangle\right\}_{l}$. Note that $\left\{\left|\phi_{x, l}\right\rangle\right\}_{l}$ is an orthonormal basis for $H_{x}$. Let $R_{x}$ be the projection onto $H_{x}$. Since we allow a little error in the decoding process, the different spaces $H_{x}$ may not be orthogonal.

Let $H$ be the space spanned by all the vectors $\left\{\left|\phi_{x, l}\right\rangle\right\}_{x, l}$, and $Q$ the projection operator onto $H$. For each $x$, let the set $\left\{\left|e_{x, j}\right\rangle\right\}_{j}$ be an orthonormal basis for the range of $P_{x}$. Then $\left\{\left|e_{x, j}\right\rangle\right\}_{x, j}$ is an orthonormal basis for the entire decoding space.

Now,

$$
\begin{aligned}
\sum_{l} \| P_{x}\left|\phi_{x, l}\right\rangle \|^{2} & =\sum_{l, j}\left|\left\langle e_{x, j} \mid \phi_{x, l}\right\rangle\right|^{2} \\
& =\sum_{j} \| R_{x}\left|e_{x, j}\right\rangle \|^{2} \\
& \leq \sum_{j} \| Q\left|e_{x, j}\right\rangle \|^{2},
\end{aligned}
$$

since the length of the projection of $\left|e_{x, j}\right\rangle$ onto $H_{x}$ is at most the length of its projection on the space $H$ (of which $H_{x}$ is a subspace).

From equation (3),

$$
\begin{aligned}
\sum_{x, l} \| P_{x}\left|\phi_{x, l}\right\rangle \|^{2} & \leq \sum_{x, j} \| Q\left|e_{x, j}\right\rangle \|^{2} \\
& =\sum_{x, j}\left\langle e_{x, j}|Q| e_{x, j}\right\rangle \\
& =\operatorname{Tr} Q=\operatorname{dim} H \\
& \leq 2^{E+m},
\end{aligned}
$$

since the space $H$ is generated by states over $E+m$ qubits.
Combining equations (1), (2), and (4), we get

$$
\operatorname{Pr}[C] \leq \frac{2^{E+m}}{2^{E-m} 2^{n}}=\frac{2^{2 m}}{2^{n}},
$$

as claimed.

Encoding with EPR pairs and ancilla leads to states very similar to those in Lemma 3.2, and Theorem 3.3 holds in that case as well. We will however skip ahead to encoding where Alice uses extra space, and an arbitrary entangled state.

\subsection{Encoding with general prior entanglement}

In general, in trying to transmit information, Alice and Bob may share an arbitrary entangled state (independent of their inputs) before they interact. In this section we show that the results in the previous section apply irrespective of which initial entangled state Alice and Bob share.

The main difficulty here is that the property of messages encoded over EPR pairs embodied in Lemma 3.2 may fail to hold. However, we show a simple connection between encoding with EPR pairs and encoding with an arbitrary entangled state that allows us to conclude an identical result.

We start by observing that we need only consider protocols which make use of a special kind of shared state.

OBSERVATION 3.4. In any quantum communication protocol with prior entanglement, we may assume, without loss of generality, that the initial shared state is of the form

$$
\sum_{a \in\{0,1\}^{E}} \sqrt{\lambda_{a}}|a\rangle_{A}|a\rangle_{B},
$$

where $\lambda_{a}$ are non-negative reals, and $\sum_{a} \lambda_{a}=1$.

Proof. This follows directly from the Schmidt decomposition theorem (Theorem 2.1). Consider a protocol $\mathcal{P}$ in which the quantum state shared by Alice and Bob has $E_{A}$ qubits on Alice's side and $E_{B}$ qubits on Bob's side. For concreteness, assume that $E_{A} \leq E_{B}$. By Theorem 2.1, the shared state may be expressed as

$$
\sum_{b \in\{0,1\}^{E_{A}}} \sqrt{\mu_{b}}\left|\phi_{b}\right\rangle_{A}\left|\psi_{b}\right\rangle_{B},
$$

where the $\mu_{b}$ are non-negative reals summing up to 1 , and the sets $\left\{\left|\phi_{b}\right\rangle\right\}$ and $\left\{\left|\psi_{b}\right\rangle\right\}$ are orthonormal. We may modify the protocol to a new protocol $\mathcal{P}^{\prime}$, which has the same behaviour as $\mathcal{P}$ on each input, but where the shared state is of the form in stated in the observation above. Consider any unitary transformations $U, V$ on $E=E_{B}$ qubits such that for every $b \in\{0,1\}^{E_{A}}$,

$$
\begin{aligned}
& U \quad: \quad|\overline{0}, b\rangle \mapsto|\overline{0}\rangle\left|\phi_{b}\right\rangle \\
& V \quad: \quad|\overline{0}, b\rangle \mapsto\left|\psi_{b}\right\rangle .
\end{aligned}
$$

Let $\lambda_{\overline{0} b}=\mu_{b}$, for $b$ as above, and let the rest of the $\lambda_{a}$ be 0 . The protocol $\mathcal{P}^{\prime}$ begins with the shared state

$$
\sum_{a \in\{0,1\}^{E}} \sqrt{\lambda_{a}}|a\rangle_{A}|a\rangle_{B},
$$

and then Alice and Bob apply $U$ and $V$ to their qubits respectively. Thereafter, the protocol proceeds exactly as in $\mathcal{P}$. By construction, the protocols behave the same way for each input. 
We make another simplifying observation about the protocols that we need consider.

OBSERVATION 3.5. In any quantum communication protocol with prior entanglement, we may assume, without loss of generality, that neither Alice nor Bob uses any ancillary qubits in their local unitary operations or measurements.

This is because all the ancillary qubits used may be considered as part of the initial shared state.

The above observations allow us to relate the encoding with a general entangled state to the encoding obtained when EPR pairs are used instead.

Lemma 3.6. Suppose that Alice performs a unitary transformation on her share of the joint state

$$
\sum_{a \in\{0,1\}^{E}} \sqrt{\lambda_{a}}|a\rangle_{A}|a\rangle_{B},
$$

and then sends $m$ of the $E$ qubits to Bob. Then, Bob has E+ $m$ qubits in a mixed state that can be represented as

$$
\left\{2^{m / 2}\left(I_{m} \otimes \Lambda\right)\left|\phi_{l}\right\rangle\right\}_{l \in\{0,1\}^{E-m}}
$$

with $\left\{\left|\phi_{l}\right\rangle\right\}_{l}$ orthonormal, and $\Lambda=\sum_{a} \sqrt{\lambda_{a}}|a\rangle\langle a|$.

Proof. Note that the shared state may be written as

$$
\left(I_{E} \otimes \Lambda\right) \sum_{a}|a\rangle|a\rangle
$$

Suppose Alice applies the transformation $V$ to her $E$ qubits. The resulting joint state is

$$
\begin{aligned}
(V & \left.\otimes I_{E}\right)\left(I_{E} \otimes \Lambda\right) \sum_{a}|a\rangle|a\rangle \\
& =\left(I_{E} \otimes \Lambda\right) \sum_{a} V|a\rangle|a\rangle \\
& =\left(I_{E} \otimes \Lambda\right) \sum_{a}|a\rangle V^{\top}|a\rangle \\
& =\sum_{l \in\{0,1\}^{E-m}}|l\rangle\left(I_{m} \otimes \Lambda\right) \sum_{r \in\{0,1\}^{m}}|r\rangle V^{\top}|l r\rangle,
\end{aligned}
$$

where equation (19) follows from Lemma 3.1. Let, as in Lemma 3.2,

$$
\left|\phi_{l}\right\rangle=2^{-m / 2} \sum_{r \in\{0,1\}^{m}}|r\rangle V^{\top}|l r\rangle .
$$

Suppose Alice sends $m$ of her qubits to Bob, and measures the remaining qubits in the standard basis. The residual, unnormalised, state with Bob is then $2^{m / 2}\left(I_{m} \otimes \Lambda\right)\left|\phi_{l}\right\rangle$, when she observes $l \in\{0,1\}^{E-m}$. That the states $\left|\phi_{l}\right\rangle$ are orthonormal is shown in the proof of Lemma 3.2.

We can now prove the equivalent of Theorem 3.3 when Alice and Bob share an arbitrary entangled state.

THEOREM 3.7. If Alice encodes $2^{n}$ messages over her part of an arbitrary (but fixed) shared entangled state and some ancillary qubits, and sends $m$ qubits to Bob, the probability of correct decoding of a message chosen uniformly at random is bounded as $\operatorname{Pr}[$ correct decoding $] \leq \frac{2^{2 m}}{2^{n}}$.
Proof. We use the same notation as in the proof of Theorem 3.3, adapted to the different encoding we get here due to the more general entangled state.

By Observation 3.5, we may assume that Alice and Bob operate only on their shared entangled state. We may further assume that this state is of the special form described in Observation 3.4 .

Let $\left\{2^{m / 2}\left(I_{m} \otimes \Lambda\right)\left|\phi_{x, l}\right\rangle\right\}$ be Bob's mixed state when Alice encodes $x \in\{0,1\}^{n}$, as given by Lemma 3.6. Since no ancilla is used in the decoding procedure (i.e., in Bob's measurement to extract $x$, cf. Observation 3.5), the projection operators $P_{y}$ are over $E+m$ qubits. Now,

$$
\begin{aligned}
\operatorname{Pr}[C] & =\sum_{x} \frac{\operatorname{Pr}\left[C_{x}\right]}{2^{n}}, \quad \text { and } \\
\operatorname{Pr}\left[C_{x}\right] & =2^{m} \sum_{l} \| P_{x}\left(I_{m} \otimes \Lambda\right)\left|\phi_{x, l}\right\rangle \|^{2} .
\end{aligned}
$$

Furthermore,

$$
\begin{aligned}
\sum_{x, l} \| P_{x}\left(I_{m} \otimes \Lambda\right)\left|\phi_{x, l}\right\rangle \|^{2} & =\sum_{x, l, j}\left|\left\langle e_{x, j}\left|\left(I_{m} \otimes \Lambda\right)\right| \phi_{x, l}\right\rangle\right|^{2} \\
& =\sum_{x, j} \| R_{x}\left(I_{m} \otimes \Lambda\right)\left|e_{x, j}\right\rangle \|^{2} \\
& \leq \sum_{x, j} \|\left(I_{m} \otimes \Lambda\right)\left|e_{x, j}\right\rangle \|^{2} \\
& =\sum_{x, j}\left\langle e_{x, j}\left|\left(I_{m} \otimes \Lambda^{2}\right)\right| e_{x, j}\right\rangle \\
& =\operatorname{Tr}\left(I_{m} \otimes \Lambda^{2}\right) \\
& =2^{m} \sum_{a} \lambda_{a}=2^{m}
\end{aligned}
$$

Combining equations (6), (4) and (8), we get

$$
\operatorname{Pr}[C] \leq 2^{2 m} / 2^{n} \text {. }
$$

\section{EXTENSION TO INTERACTIVE COMMUNICATION}

\subsection{The main lemma}

In this section, we analyse the most general quantum protocols for exchanging information. In these protocols, Alice and Bob share an arbitrary entangled state to begin with, and exchange messages both ways in order to communicate.

The essential idea behind the results below is contained in Lemma 3.6, and leads to a new characterisation of the joint state in quantum protocols. In order to prove the lemma from first principles, we focus on protocols in which there is no prior entanglement. That it holds also for communication with prior entanglement may be inferred from the lemma itself by applying it to a protocol in which the prior shared entanglement is generated by Bob creating the state to be shared, and sending the appropriate part of it to Alice.

Lemma 4.1. Let $\mathcal{P}$ be any quantum communication protocol (without prior entanglement) in which the number of qubits sent by Alice to Bob (Bob to Alice) is $m_{A}$ (respectively, $m_{B}$ ), and the final number of qubits with Alice (Bob) is $q_{A}$ (respectively, $q_{B}$ ). Then, the joint state of Alice and 
Bob at the end of the protocol may be expressed as

$$
\sum_{a \in\{0,1\}^{q} A}|a\rangle_{A} \Lambda\left|\phi_{a}\right\rangle_{B}
$$

where

1. $\Lambda$ is a linear transformation that maps $q_{B}+2 m_{B}$ qubits to $q_{B}$ qubits, depends only on the unitary transformations of Bob, and satisfies $\operatorname{Tr}\left(\Lambda \Lambda^{\dagger}\right)=2^{2 m_{A}}$, and

2. $\left\{\left|\phi_{a}\right\rangle\right\}$ is an orthonormal set of states over $q_{B}+2 m_{B}$ qubits, and depends only on the unitary transformations of Alice.

Proof. The proof goes by induction on the number of rounds $t$.

In the beginning (for $t=0$ ), the joint state (w.l.o.g.) is $|\overline{0}\rangle_{A} \otimes|\overline{0}\rangle_{B}$, which represents all the qubits the two players use during the protocol. This is of the form described in the lemma, with $\Lambda=\Lambda_{0}=|\overline{0}\rangle\langle\overline{0}|$.

Let $q_{A, t}, q_{B, t}, m_{A, t}, m_{B, t}$ be the quantities corresponding to $q_{A}, q_{B}, m_{A}, m_{B}$ after $t \geq 0$ rounds of communication. Assume that at this stage, the joint state of Alice and Bob is

$$
\sum_{a \in\{0,1\}^{q_{A, t}}}|a\rangle_{A} \Lambda_{t}\left|\phi_{a, t}\right\rangle_{B}
$$

where $\Lambda_{t}$ and $\left\{\left|\phi_{a, t}\right\rangle\right\}$ satisfy the conditions stated in the lemma (in terms of $q_{B, t}, m_{A, t}, m_{B, t}$ ). We look at two cases for the $(t+1)$ 'th round of communication.

CASE (a). Alice applies a unitary transformation $U$ to her qubits and sends $p$ qubits to Bob.

The state after the unitary transformation is

$$
\begin{aligned}
(U & \left.\otimes I_{q_{B, t}}\right)\left(I_{q_{A, t}} \otimes \Lambda_{t}\right) \sum_{a \in\{0,1\}^{q_{A, t}}}|a\rangle\left|\phi_{a, t}\right\rangle \\
& =\left(I \otimes \Lambda_{t}\right) \sum_{a \in\{0,1\}^{q_{A, t}}} U|a\rangle\left|\phi_{a, t}\right\rangle \\
& =\left(I \otimes \Lambda_{t}\right) \sum_{a \in\{0,1\}^{q_{A, t}}}|a\rangle \tilde{U}\left|\phi_{a, t}\right\rangle,
\end{aligned}
$$

where $\tilde{U}$ is a unitary transformation on Bob's qubits as given by Lemma 3.1. Thus, after Alice sends $p$ of her qubits to Bob (w.l.o.g., these are the $p$ rightmost qubits), the joint state looks like

$$
\sum_{l \in\{0,1\}^{q_{A, t+1}}}|l\rangle_{A}\left(I_{p} \otimes \Lambda_{t}\right) \sum_{r \in\{0,1\}^{p}}|r\rangle_{B} \tilde{U}\left|\phi_{l r, t}\right\rangle_{B} .
$$

Here, $q_{A, t+1}=q_{A, t}-p, q_{B, t+1}=q_{B, t}+p, m_{A, t+1}=m_{A, t}+p$, and $m_{B, t+1}=m_{B, t}$.

Let

$$
\begin{aligned}
\Lambda_{t+1} & =2^{p / 2}\left(I_{p} \otimes \Lambda_{t}\right), \quad \text { and } \\
\left|\phi_{l, t+1}\right\rangle & =2^{-p / 2} \sum_{r}|r\rangle \tilde{U}\left|\phi_{l r, t}\right\rangle .
\end{aligned}
$$

Now,

$$
\begin{aligned}
\operatorname{Tr} \Lambda_{t+1} \Lambda_{t+1}^{\dagger} & =2^{p} \operatorname{Tr}\left(I_{p} \otimes \Lambda_{t} \Lambda_{t}^{\dagger}\right) \\
& =2^{p} \cdot 2^{p} \cdot 2^{2 m_{A, t}} \\
& =2^{2 m_{A, t+1}}
\end{aligned}
$$

Moreover, for the same reasons as in the proof of Lemma 3.2, the set $\left\{\left|\phi_{l, t+1}\right\rangle\right\}$ is orthonormal. Thus, the state in equation (9) is of the form stated in the lemma.

CASE (b). Bob applies a unitary transformation $V$ to his qubits and sends $p$ qubits to Alice. W.l.o.g., these are the $p$ leftmost qubits.

After the communication, the joint state looks like

$$
\sum_{a \in\{0,1\}^{q_{A}, t}} \sum_{l \in\{0,1\}^{p}}|a\rangle_{A}|l\rangle_{A} \quad\left(\langle l| \otimes I_{q_{B, t}-p}\right) V \Lambda_{t}\left|\phi_{a, t}\right\rangle_{B},
$$

which may be recast as

$$
\sum_{a, l}|a l\rangle \Lambda_{t+1}\left|\phi_{a l, t+1}\right\rangle
$$

where

$$
\begin{aligned}
\Lambda_{t+1} & =\sum_{b \in\{0,1\}^{p}}\left(\langle b| \otimes I_{q_{B, t}-p}\right) V \Lambda_{t}\left(\langle b| \otimes I_{q_{B, t}}\right), \\
\left|\phi_{a l, t+1}\right\rangle & =|l\rangle\left|\phi_{a, t}\right\rangle .
\end{aligned}
$$

Now $m_{A, t+1}=m_{A, t}, m_{B, t+1}=m_{B, t}+p, q_{A, t+1}=q_{A, t}+p$, and $q_{B, t+1}=q_{B, t}-p$.

The states $\left|\phi_{a l, t+1}\right\rangle$ are orthonormal. Moreover,

$$
\begin{aligned}
\operatorname{Tr} & \Lambda_{t+1} \Lambda_{t+1}^{\dagger} \\
& =\operatorname{Tr} \sum_{b, b^{\prime}}(\langle b| \otimes I) V \Lambda_{t}(\langle b| \otimes I)\left(\left|b^{\prime}\right\rangle \otimes I\right) \Lambda_{t}^{\dagger} V^{\dagger}(|b\rangle \otimes I) \\
& =\sum_{b} \operatorname{Tr}\left[(\langle b| \otimes I) V \Lambda_{t} \Lambda_{t}^{\dagger} V^{\dagger}(|b\rangle \otimes I)\right] \\
& =\sum_{b} \operatorname{Tr}\left[(|b\rangle\langle b| \otimes I) V \Lambda_{t} \Lambda_{t}^{\dagger} V^{\dagger}\right] \\
& =\operatorname{Tr}\left[(I \otimes I) V \Lambda_{t} \Lambda_{t}^{\dagger} V^{\dagger}\right] \\
& =\operatorname{Tr} \Lambda_{t} \Lambda_{t}^{\dagger}=2^{2 m_{A, t+1}} .
\end{aligned}
$$

Thus, the state in equation (10) is of the form described in the lemma.

This completes the induction step, and the proof.

\subsection{Implications for communication problems}

We now sketch how our characterisation of quantum protocols enables us to prove Theorem 1.1.

Proof of TheOrEm 1.1. The proof is essentially the same as for Theorem 3.7, and we use the same notation here.

Lemma 4.1 shows that Bob's state remains of form similar to that in Lemma 3.6 as he interacts with Alice during the protocol. Let $\left\{\Lambda\left|\phi_{x, l}\right\rangle\right\}_{l}$ be Bob's mixed state at the end of the protocol when Alice has input $x \in\{0,1\}^{n}$, as given by Lemma 4.1. Note that $\Lambda$ is independent of $x$.

Since we may assume that all the ancillary qubits used by Bob are included in his state above (cf. the proof of Lemma 4.1), the projection operators $P_{y}$ are over $q_{B}$ qubits. Now, as before,

$$
\operatorname{Pr}[C]=\sum_{x} \frac{\operatorname{Pr}\left[C_{x}\right]}{2^{n}}=\frac{1}{2^{n}} \sum_{x, l} \| P_{x} \Lambda\left|\phi_{x, l}\right\rangle \|^{2} .
$$


Furthermore,

$$
\begin{aligned}
\sum_{x, l} \| P_{x} \Lambda\left|\phi_{x, l}\right\rangle \|^{2} & =\sum_{x, l, j}\left|\left\langle e_{x, j}|\Lambda| \phi_{x, l}\right\rangle\right|^{2} \\
& \leq \sum_{x, j} \| \Lambda^{\dagger}\left|e_{x, j}\right\rangle \|^{2} \\
& =\operatorname{Tr} \Lambda \Lambda^{\dagger}=2^{2 m_{A}}
\end{aligned}
$$

Combining these, we get $\operatorname{Pr}[C] \leq 2^{2 m_{A}} / 2^{n}$.

Finally, we apply Theorem 1.1 to obtain an improved lower bound for the entanglement-assisted quantum communication complexity of the inner product function $\mathrm{IP}_{n}$. (The function $\operatorname{IP}_{n}:\{0,1\}^{n} \times\{0,1\}^{n} \rightarrow\{0,1\}$ is defined as $\operatorname{IP}_{n}(x, y)=\oplus_{i}\left(x_{i} \wedge y_{i}\right)$.) The connection between the two is provided by the following reduction due to Cleve et al. [8].

Theorem 4.2 (Cleve, van Dam, Nielsen, Tapp). If $Q_{\epsilon}^{*}\left(\mathrm{IP}_{n}\right)=m$, then there is an entanglement-assisted protocol for transmitting $n$ bits with probability of success at least $(1-2 \epsilon)^{2}$, such that the total communication from each party to the other, over all the rounds of communication, is $m$ qubits.

Theorem 1.1 now implies

$$
\text { Corollary 4.3. } Q_{\epsilon}^{*}\left(\operatorname{IP}_{n}\right) \geq \frac{1}{2}\left(n-\log \frac{1}{(1-2 \epsilon)^{2}}\right) .
$$

It is not hard to see that for any $\epsilon<1 / 2$, there is a publiccoin randomised protocol for $\mathrm{IP}_{n}$ with communication cost at most $n-\log \frac{1}{1-2 \epsilon}+1$. Along with the superdense coding scheme of [4], this means that

Theorem 4.4. $Q_{\epsilon}^{*}\left(\operatorname{IP}_{n}\right) \leq \frac{1}{2}\left(n-\log \frac{1}{1-2 \epsilon}+1\right)$.

Thus, our lower bound is close to optimal, and for constant error, is within an additive $O(1)$ term of the upper bound. Since $Q_{1 / 3}\left(\mathrm{IP}_{n}\right) \leq n$, this provides more evidence that prior entanglement does not give us a saving of more than a factor 2 (plus perhaps an additive term of $O(\log n)$ ) in communication cost.

\section{ACKNOWLEDGEMENTS}

We would like to thank Leonard Schulman for insightful discussions, and Umesh Vazirani and the anonymous referees for helpful comments on the paper.

\section{REFERENCES}

[1] A. Ambainis, L. J. Schulman, A. Ta-Shma, U. Vazirani, and A. Wigderson. The quantum communication complexity of sampling. In Proceedings of the 39th Annual IEEE Symposium on Foundations of Computer Science, pages 342-351, 1998.

[2] C. Bennett, C. Crépeau, R. Jozsa, A. Peres, and W. Wootters. Teleporting an unknown quantum state via dual classical and Einstein-Podolsky-Rosen channels. Physical Review Letters, 70:1895-1899, 1993.

[3] C. Bennett and P. Shor. Quantum information theory. IEEE Transactions on Information Theory, IT-44(6):2724-2742, 1998.

[4] C. Bennett and S. Wiesner. Communication via oneand two-particle operators on Einstein-Podolsky-Rosen states. Physical Review Letters, 69:2881-2884, 1992.
[5] E. Bernstein and U. Vazirani. Quantum complexity theory. SIAM Journal on Computing, 26(5):1411-1473, 1997.

[6] H. Buhrman, R. Cleve, and A. Wigderson. Quantum vs. classical communication and computation. In Proceedings of the 30th Annual ACM Symposium on Theory of Computing, pages 63-68, 1998.

[7] H. Buhrman and R. de Wolf. Communication complexity lower bounds by polynomials. In Proceedings of the 16th Annual IEEE Conference on Computational Complexity, pages 120-130, 2001.

[8] R. Cleve, W. van Dam, M. Nielsen, and A. Tapp. Quantum entanglement and the communication complexity of the inner product function. In Quantum Computing and Quantum Communications, Proceedings of the 1st NASA International Conference, volume 1509 of Lecture Notes in Computer Science, pages 61-74, 1998.

[9] T. M. Cover and J. A. Thomas. Elements of Information Theory. Wiley Series in

Telecommunications. John Wiley \& Sons, New York, NY, USA, 1991.

[10] A. Holevo. Some estimates of the information transmitted by quantum communication channels. Problems of Information Transmission, 9:177-183, 1973.

[11] H. Klauck. On quantum and probabilistic communication: Las Vegas and one-way protocols. In Proceedings of the 32nd Annual ACM Symposium on Theory of Computing, pages 644-651, 2000.

[12] H. Klauck. Lower bounds for quantum communication complexity. In Proceedings of the 42nd Annual IEEE Symposium on Foundations of Computer Science, pages 288-297, 2001.

[13] H. Klauck, A. Nayak, A. Ta-Shma, and D. Zuckerman. Interaction in quantum communication and the complexity of Set Disjointness. In Proceedings of the 33rd Annual ACM Symposium on Theory of Computing, pages 124-133, 2001.

[14] E. Kushilevitz and N. Nisan. Communication Complexity. Cambridge University Press, 1997.

[15] A. Nayak. Lower Bounds for Quantum Computation and Communication. PhD thesis, University of California, Berkeley, 1999.

[16] A. Nayak. Optimal lower bounds for quantum automata and random access codes. In Proceedings of the 40th Annual IEEE Symposium on Foundations of Computer Science, pages 369-376, 1999.

[17] M. A. Nielsen and I. L. Chuang. Quantum Computation and Quantum Information. Cambridge University Press, 2000.

[18] R. Raz. Exponential separation of quantum and classical communication complexity. In Proceedings of the 31st Annual ACM Symposium on Theory of Computing, pages 358-367, 1999.

[19] W. van Dam and P. Hayden. Communication complexity of quantum state transformations. Manuscript, 2001.

[20] A. C.-C. Yao. Quantum circuit complexity. In Proceedings of the 34th Annual IEEE Symposium on Foundations of Computer Science, pages 352-361, 1993. 